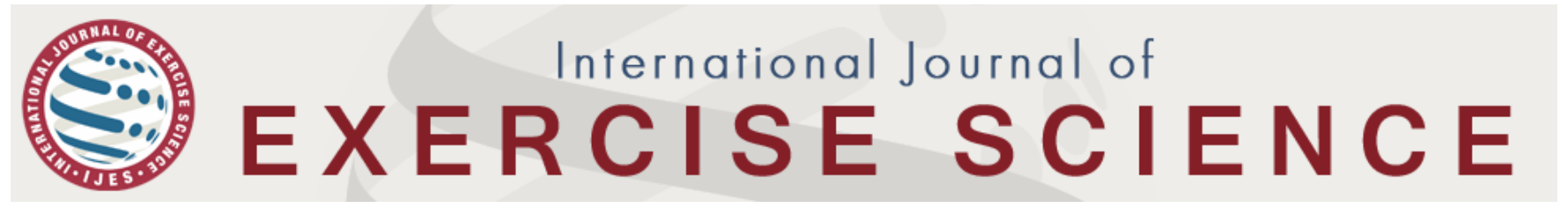

Original Research

\title{
Interlimb Asymmetry Thresholds that Negatively Affect Change of Direction Performance in Collegiate American Football Players
}

\author{
NICOLAS M. PHILIPP $¥ 1$, DEREK A. CRAWFORD $¥ 1$, MATTHEW J. GARVER $¥ 1$, DUSTIN W. \\ DAVIS ${ }^{2}$, and JOSIE N. HAIR ${ }^{\dagger 1}$
}

1School of Nutrition, Kinesiology and Psychological Science, University of Central Missouri, Warrensburg, MO, USA; ${ }^{2}$ Department of Kinesiology and Nutrition Sciences, University of Nevada, Las Vegas, Las Vegas, NV, USA

†Denotes graduate student author, $¥$ Denotes professional author

\begin{abstract}
International Journal of Exercise Science 14(4): 606-612, 2021. Researchers and strength and conditioning practitioners have had an increased interest in the effects of interlimb asymmetries on different aspects of sport performance over the past couple of years. Interlimb asymmetries have been found to negatively affect performance in key performance indicators (KPI) such as jumping, sprinting, and changing directions, within various sports populations. However, there is no consensus about a meaningful threshold at which asymmetries start to negatively affect KPIs or performance. The aim of this study was to investigate a potentially meaningful threshold for three asymmetry metrics (mean peak velocity [mPV], mean peak power [mPP], mean average power $[\mathrm{mAP}])$ that were extracted from the Bulgarian split squat and found to be significantly related to change of direction performance (via L-drill test) within a sample of collegiate American football players. Receiver operating characteristic (ROC) curves were used to identify asymmetry thresholds for all metrics that discriminated between faster and slower performers in the L-drill. Players with asymmetries over 10.65\% (mAP), 14.59\% (MPP), and $14.96 \%(\mathrm{mPV})$ were identified by ROC curves as more likely to be classified as low performers. These findings may be helpful for practitioners interested in screening athletes for interlimb asymmetries that may negatively affect their change of direction performance.
\end{abstract}

KEY WORDS: Asymmetry, athlete monitoring, threshold, sports science, single leg

\section{INTRODUCTION}

Interest in the effects of interlimb asymmetry, with respect to successful sports performance, has increased in recent years. Some literature highlights associations between lower extremity neuromuscular function (e.g., unilateral power production capability) and performance in sport-related tasks such as jumping (2), sprinting (3), and change of direction (CoD) $(2,3,8)$. Simultaneously, literature also attests that there is not a clear understanding of the influence of asymmetries on athletic performance measures (9). 
Research focused on the magnitude of asymmetry that may negatively affect sports performance is lacking. Within the realms of return-to play research, specifically following ACL-ruptures, several studies have proposed asymmetry thresholds ranging from $10-15 \%$ as potentially meaningful interlimb differences $(5,7,11,13)$. Athletes reporting asymmetries below these thresholds were considered safe to return to action. Further, Knapik et al. have proposed that asymmetries under $15 \%$ may not be functionally significant when using the difference to detect risk for potential injury (6). However, these works do not focus on meaningful asymmetry thresholds that affect performance. In previous work, we have suggested that identifying a threshold of asymmetry that impacts sports performance is important to practitioners looking to reduce asymmetries and their effects. Identification would provide evidence-based targets for interventions (12).

Examining the effects of asymmetry directly connected to key performance indicators (KPI) is needed. To the best of our knowledge, no evidence-based threshold of interlimb asymmetry that has meaningful effects on $\mathrm{CoD}$ performance has been put forth. Extending on our previous work (12), this study identifies a meaningful threshold for various interlimb asymmetry metrics (e.g., lower body power) that are associated with CoD performance assessed via the L-drill in collegiate American football players.

\section{METHODS}

\section{Participants}

A sample of 24 skill position players (age $=19.8 \pm 0.9$ years; height $=179.0 \pm 3.4 \mathrm{~cm}$; mass $=83.2 \pm 5.7$ $\mathrm{kg} ; \mathrm{n}=19$ for body fat $=9.9 \pm 3.1 \%$; lean mass $=43.6 \pm 2.9 \mathrm{~kg}$ ) participated in all study procedures.

\section{Protocol}

This is a secondary analysis of a previous study demonstrating an association between interlimb asymmetry and performance in Combine-related tests among collegiate American Football players (12). All study procedures were approved by the University Institutional Review Board in accordance with the Declaration of Helsinki. Further, this research was carried out fully in accordance to the ethical standards of the International Journal of Exercise Science (10). All details, protocols, and data treatment can be found within the original investigation (12). This analysis only includes asymmetry metrics that significantly correlated with L-drill performance. The L-drill is a CoD assessment commonly used within this specific population and it was selected based on its' usage within the NFL combine (12).

Volunteers completed two testing sessions. The first session included reading and signing of the informed consent. Thereafter, collection of anthropometric data (e.g., height and weight) and the performance of unilateral countermovement jumps (CMJ) and a three-repetition maximal Bulgarian split squat (3 RM BSS) task were completed. The second session took place during the University football team's annual "Spring Testing" day. A team-led, standardized warm-up was employed. Volunteers then completed the Combine-related testing drills (e.g., L-drill, broad jump, and vertical jump). 
Asymmetry Assessment and Classification: Mean peak velocity (mPV), mean peak power $(\mathrm{mPP})$, and mean average power (mAP) were extracted from the final successful lift for each limb. Asymmetry was calculated using the following formula: [(max value - min value)/max value] $x$ 100. Post hoc receiver operator characteristic (ROC) curve analysis revealed that greater asymmetry (i.e., asymmetry over threshold) is less desirable and related to slower L-drill performance, while a lower asymmetry (i.e., asymmetry under threshold) is more desirable, reflecting a faster L-drill performance.

Change of Direction Performance and Classification: CoD was assessed with the L-drill. The fastest time of three attempts was used for analysis. Time was used to classify "slower" and "faster" CoD performers within the L-drill task. The $50^{\text {th }}$ percentile value of $6.97 \mathrm{sec}$ established the discriminating point between faster (i.e., $\leq 6.97 \mathrm{sec}$ ) and slower (i.e., $>6.97 \mathrm{sec}$ ) performers. Using this method, players classified as faster $(\mathrm{n}=13 ; 6.80 \pm 0.12 \mathrm{sec})$ were significantly faster (mean difference $=-0.34 ; 95 \% \mathrm{CI}=-0.45,-0.23 \mathrm{sec} ; \mathrm{t}=-6.20, \mathrm{p}<.001)$ than the slower $(\mathrm{n}=11$; $7.14 \pm 0.14 \mathrm{sec}$ ) performers.

\section{Statistical Analysis}

We used ROC curve analyses for each asymmetry metric $-\mathrm{mPV}, \mathrm{mPP}$, and $\mathrm{mAP}-$ to establish cut-off scores (i.e., thresholds) that maximized the positive predictive value (i.e., sensitivity) and minimized the false positive rate (i.e., 1-specificity) associated with slower and faster performers. Using the identified cut-off scores from the ROC curve analyses, $2 \times 2$ tables were constructed to develop estimates of diagnostic utility (e.g., validity and reliability). All analyses were conducted using SPSS version 24.0 (IBM, Inc., Armonk, NY), and an a level of 0.05 was used for statistical inferences.

\section{RESULTS}

The ROC analyses revealed that asymmetry metrics ranging from $10.65 \%$ (mAP) to $14.96 \%$ $(\mathrm{mPV})$ were able to discriminate L-drill performance, but with notably different diagnostic utility. Figure 1 shows the individual ROC curves for each metric, and Table 1 highlights asymmetry classifications, CoD classifications, and resulting diagnostic utility when using $\mathrm{mPV}, \mathrm{mPP}$, and $\mathrm{mAP}$ as screening tools.

A significant ROC curve (AUC $=.811,95 \% \mathrm{CI}=.634-.989 ; \mathrm{p}=.010)$ produced a threshold score (i.e., $14.96 \%$ ) for $\mathrm{mPV}$ asymmetry that was able to correctly classify 9 out of 11 players $(82 \%)$ who were slower performers and 9 out of $13(69 \%)$ who were faster performers. A significant ROC curve ( $\mathrm{AUC}=.797,95 \% \mathrm{CI}=.614-.981 ; \mathrm{p}=.014)$ produced a threshold score (i.e., $14.59 \%$ ) for $\mathrm{mPP}$ asymmetry that was able to correctly classify 9 out of 11 players $(82 \%)$ who were slower performers and 9 out of $13(69 \%)$ who were faster performers. A significant ROC curve (AUC = $.741,95 \% \mathrm{CV}=.533-.950 ; \mathrm{p}=.046)$ produced a threshold score (i.e., $10.65 \%$ ) for $\mathrm{mAP}$ asymmetry that was able to correctly classify 8 out of 10 players (80\%) who were slower performers and 9 out of $14(62 \%)$ who were faster performers. 

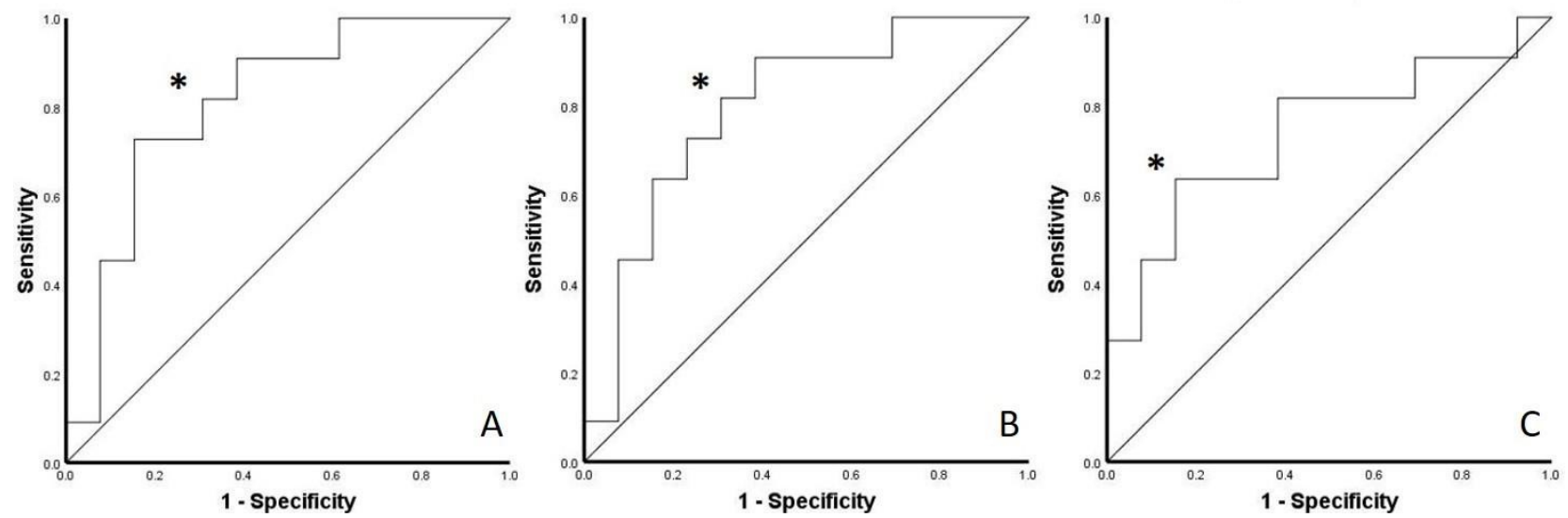

Figure 1. Receiver operating characteristic (ROC) curves for L-drill performance classification and asymmetry in (a) mean peak velocity, (b) mean peak power, and (c) mean average power. ${ }^{*}=$ Point on the curve that maximizes sensitivity and specificity.

Table 1. 2x2 summary table of asymmetry threshold screening and CoD performance.

\begin{tabular}{|c|c|c|c|c|c|c|}
\hline \multirow[b]{3}{*}{ CoD Classification } & \multicolumn{2}{|c|}{ Mean Peak Velocity } & \multicolumn{2}{|c|}{ Mean Peak Power } & \multicolumn{2}{|c|}{ Mean Average Power } \\
\hline & \multicolumn{6}{|c|}{ Asymmetry Classification } \\
\hline & $\geq 14.96 \%$ & $<14.96 \%$ & $>14.59 \%$ & $<14.59 \%$ & $\geq 10.65 \%$ & $<10.65 \%$ \\
\hline Slower & 9 & 2 & 9 & 2 & 8 & 2 \\
\hline Faster & 4 & 9 & 4 & 9 & 5 & 9 \\
\hline \multicolumn{7}{|l|}{ Diagnostic Utility } \\
\hline Diagnostic Accuracy & \multicolumn{2}{|c|}{$75 \%$} & \multicolumn{2}{|c|}{$75 \%$} & \multicolumn{2}{|c|}{$71 \%$} \\
\hline Sensitivity & \multicolumn{2}{|c|}{$69 \%$} & \multicolumn{2}{|c|}{$69 \%$} & \multicolumn{2}{|c|}{$62 \%$} \\
\hline Specificity & \multicolumn{2}{|c|}{$82 \%$} & \multicolumn{2}{|c|}{$82 \%$} & \multicolumn{2}{|c|}{$80 \%$} \\
\hline False Positive Rate & \multicolumn{2}{|c|}{$31 \%$} & \multicolumn{2}{|c|}{$31 \%$} & \multicolumn{2}{|c|}{$38 \%$} \\
\hline False Negative Rate & \multicolumn{2}{|c|}{$18 \%$} & \multicolumn{2}{|c|}{$18 \%$} & \multicolumn{2}{|c|}{$18 \%$} \\
\hline Positive Predictive Value & \multicolumn{2}{|c|}{$82 \%$} & \multicolumn{2}{|c|}{$82 \%$} & \multicolumn{2}{|c|}{$80 \%$} \\
\hline Negative Predictive Value & \multicolumn{2}{|c|}{$69 \%$} & \multicolumn{2}{|c|}{$69 \%$} & \multicolumn{2}{|c|}{$64 \%$} \\
\hline Prevalence & \multicolumn{2}{|c|}{$54 \%$} & \multicolumn{2}{|c|}{$54 \%$} & \multicolumn{2}{|c|}{$54 \%$} \\
\hline Positive Likelihood Ratio & \multicolumn{2}{|c|}{2.2} & \multicolumn{2}{|c|}{2.2} & \multicolumn{2}{|c|}{1.6} \\
\hline Negative Likelihood Ratio & \multicolumn{2}{|c|}{0.2} & \multicolumn{2}{|c|}{0.2} & \multicolumn{2}{|c|}{0.2} \\
\hline
\end{tabular}

\section{DISCUSSION}

The current investigation sought to identify a meaningful threshold of interlimb asymmetry that affects CoD performance in a sample of collegiate American Football players. Our findings highlight that asymmetries in $\mathrm{mPV}, \mathrm{mPP}$, and $\mathrm{mAP}$ discriminate faster and slower L-drill 
performance. A closer look reveals the optimal cut-off score that maximized sensitivity for asymmetry in $\mathrm{mPV}$ and $\mathrm{mPP}$ was a threshold approaching $15 \%$. The optimal cut-off score that maximized sensitivity for asymmetry in mAP was a threshold nearer $10 \%$. Referring to the introduction, one can see the similarity between our asymmetry thresholds (focused on asymmetry and the KPI of $\mathrm{CoD}$ ) and the threshold suggested by Knapik et al. for detecting risk for potential injury.

To our knowledge, this is the first study within this population that sought to find answers about a meaningful interlimb asymmetry threshold that affects the KPI of CoD. Examining the measures of diagnostic utility helps better reveal which of these metrics may be used for predicting performance in the L-drill in this population. Focusing on $\mathrm{mPV}$ and $\mathrm{mPP}$, both metrics demonstrate acceptable validity with regards to sensitivity $(\mathrm{mPV}=69 \%, \mathrm{mPP}=69 \%)$ and specificity $(\mathrm{mPV}=82 \%, \mathrm{mPP}=82 \%)$. Similarly, the false positive rate $(\mathrm{mPV}=31 \%, \mathrm{mPP}=$ $31 \%)$, as well as the false negative rate $(\mathrm{mPV}=18 \%, \mathrm{mPP}=18 \%)$ also demonstrate a high degree of reliability for these asymmetry metrics. Asymmetry in the mAP showed reduced sensitivity, specificity, and increased false positive rate (Table 1). For this reason, we would not recommend the use of this metric when $\mathrm{mPV}$ and $\mathrm{mPP}$ can be captured within the same exercise test (i.e., BSS).

Another interesting observation from these data is the prevalence of potentially meaningful asymmetry among collegiate American football players. Reasons for the prevalence may stem from different sources. We could speculate that injury history, pre-existing anatomical interlimb-differences, or the novelty of the 3RM BSS task for the athletes contributed singularly, or collectively, to the increased interlimb asymmetry scores. We are not able to comment beyond this speculation. For all asymmetry metrics, the observed prevalence of meaningful (i.e., above threshold) asymmetry was $54 \%$. This finding is acutely interesting as the participants within this sample were highly trained (i.e., $\geq 2$ years in a collegiate strength and conditioning program) athletes. This raises the question as to whether traditional strength and conditioning practices sufficiently address the potential interlimb asymmetries that may be affecting $\mathrm{CoD}$ or other KPIs. This matter represents a potential opportunity to refine current strength and conditioning strategies to reduce interlimb asymmetry in collegiate American football.

Studies may productively extend this line of inquiry by establishing the meaningfulness of interlimb asymmetries in relation to test variability (i.e., coefficient of variation) with regards to different performance tasks and athletic populations as recently highlighted by Bishop and Dos'Santos et al. $(1,4)$. Future research should test interventions that help athletes attenuate interlimb asymmetries and determine if those reductions positively affect KPIs and sportspecific performance.

Practitioners may refer to the results found within this study when testing athletes for interlimb asymmetries with the goal of maximizing $\mathrm{CoD}$ performance. It is reasonable to speculate that as interlimb asymmetries in $\mathrm{mPV}$ and $\mathrm{mPP}$ from the $3 \mathrm{RM}$ BSS approach $15 \%$, there may be negative implications for $\mathrm{CoD}$ performance in collegiate football players. In addition, 
Int J Exerc Sci 14(4): 606-612, 2021

practitioners may find it valuable to use these metrics to gauge the effectiveness of interventions and monitor seasonal changes, should they occur.

In summary, asymmetry metrics extracted from the BSS are predictive of an important KPI outcome in collegiate American football players. Metrics show that differences ranging from $10.65 \%(\mathrm{mAP})$ up to $14.96 \%(\mathrm{mPV})$ were able to discriminate L-drill performance. The notable differences in diagnostic utility promote authors to highlight that asymmetries over $15 \%$ in lower limb $\mathrm{mPV}$ and $\mathrm{mPP}$ may negatively affect CoD performance within collegiate American football players.

\section{REFERENCES}

1. Bishop C. Interlimb Asymmetries: Are Thresholds a Usable Concept? Strength Cond J 43(1): 32-36, 2021.

2.Bishop C, Brashill C, Abbott W, Read P, Lake J, Turner A. Jumping Asymmetries Are Associated With Speed, Change of Direction Speed, and Jump Performance in Elite Academy Soccer Players. J Strength Cond Res Epub Ahead of Print, 2019.

3. Bishop C, Turner A, Maloney S, Lake J, Loturco I, Bromley T, et al. Drop Jump Asymmetry is Associated with Reduced Sprint and Change-of-Direction Speed Performance in Adult Female Soccer Players. Sports 7(1): $29,2019$.

4. Dos'Santos T, Thomas C, Jones PA. Assessing Interlimb Asymmetries: Are We Heading in the Right Direction? Strength Cond J Epub Ahead of Print, 2020.

5. Grindem H, Logerstedt D, Eitzen I, Moksnes H, Axe MJ, Snyder-Mackler L, et al. Single-Legged Hop Tests as Predictors of Self-Reported Knee Function in Nonoperatively Treated Individuals With Anterior Cruciate Ligament Injury. Am J Sports Med 39(11): 2347-2354, 2011.

6. Knapik JJ, Bauman CL, Jones BH, Harris JM, Vaughan L. Preseason strength and flexibility imbalances associated with athletic injuries in female collegiate athletes. Am J Sports Med 19(1): 76-81, 1991.

7. Kyritsis P, Bahr R, Landreau P, Miladi R, Witvrouw E. Likelihood of ACL graft rupture: not meeting six clinical discharge criteria before return to sport is associated with a four times greater risk of rupture. Br J Sports Med 50(15): 946-951, 2016.

8. Madruga-Parera M, Bishop C, Beato M, Fort-Vanmeerhaeghe A, Gonzalo-Skok O, Romero-Rodríguez D. Relationship Between Interlimb Asymmetries and Speed and Change of Direction Speed in Youth Handball Players. J Strength Cond Res Epub Ahead of Print, 2019.

9. Maloney SJ. The Relationship Between Asymmetry and Athletic Performance. J Strength Cond Res 33(9): 25792593, 2019.

10. Navalta JW, Stone WJ, Lyons TS. Ethical Issues Relating to Scientific Discovery in Exercise Science. Int J Exerc Sci 12(1): 1-8, 2019.

11. Noyes FR, Barber SD, Mangine RE. Abnormal lower limb symmetry determined by function hop tests after anterior cruciate ligament rupture. Am J Sports Med 19(5): 513-518, 1991.

12. Philipp NM, Garver MJ, Crawford DA, Davis DW, Hair JN. Interlimb asymmetry in collegiate American football players: Effects on combine-related performance. J Hum Sport Exerc 17(3), 2021. 
13. Rohman E, Steubs JT, Tompkins M. Changes in Involved and Uninvolved Limb Function During Rehabilitation After Anterior Cruciate Ligament Reconstruction. Am J Sports Med 43(6): 1391-1398, 2015. 